\title{
A Comparative Study between Cloud Energy Consumption Measuring Simulators
}

\author{
Muhammad Hassaan \\ Department of Computer Science, Virtual University of Pakistan
}

Received: 09 December 2019; Accepted: 14 February 2020; Published: 08 April 2020

\begin{abstract}
Cloud computing is the study of using remote servers which are hosted on the internet to deliver on-demand computing resources on pay-for-use basis rather than a local server. Due to high energy consumption in cloud computing environments, it has become the main area of research. The amount of energy consumption becomes difficult to determine in real cloud computing infrastructure. In recent years some simulation tools have been developed to analyze the energy consumption in cloud computing environments for the researchers. This paper evaluates three different popular cloud energy consumption measuring simulators: CloudSim, CloudAnalyst and GreenCloud. All of these simulators can determine energy consumption according to their ability and capacity. The comparative study indicates that in a cloud computing environment, GreenCloud simulator is better than CloudSim and CloudAnalyst due to reliable measurement of energy consumption. The significance of this comparative study is to help out the researchers who are working on energy consumption in cloud computing environments that how GreenCloud simulator make their work efficient rather than other simulators.
\end{abstract}

Index Terms: Cloud Computing; Energy Consumption; CloudSim; CloudAnalyst; GreenCloud

(C) 2020 Published by MECS Publisher. Selection and/or peer review under responsibility of the Research Association of Mode rn Education and Computer Science

\footnotetext{
* Corresponding author.

E-mail address:
} 


\section{Introduction}

According to IBM, "Cloud computing, often referred to as simply "the cloud," is the delivery of on-demand computing resources-everything from applications to data centers-over the Internet on a pay-for-use basis.” [1]. Cloud Computing refers to manipulate, configure and access the applications online. It offers online data storage, infrastructure and applications [2]. So, it has changed the method of exchanging information between people. Infrastructure as a Service (IaaS), Platform as a Service (PaaS) and Software as a Service (SaaS) are the three main services of cloud computing [3], [17]. Cloud computing empowers a number of people to utilize the offered utilities by simply utilizing their own gadgets without realizing that where these services models are deployed. It can be seen that by increasing the amount of energy consumption due to increased development of cloud computing environments, researchers are finding the solutions to reduce the amount of energy consumption. So, the complexity of these systems, become a problem for the researchers when they conduct study on the real cloud computing environments. Researchers can evaluate the energy consumption in cloud computing environments with the help of cloud energy consumption measuring simulators. How they are suitable to measure energy consumption of a cloud system is accessed [4]. However, GreenCloud is much better than other simulators due to good performance and less simulation time. It has also different power saving modes i.e. DVFS (Dynamic Voltage and Frequency Scaling) and DNS (Dynamic Network Shutdown) [14,15] which are not in other simulators. The only main limitation of GreenCloud simulator is that it limits to only small data centers [26]. In this paper, the comparative study of three different simulators which are specifically developed for cloud energy consumption measurement i.e. CloudSim; CloudAnalyst and GreenCloud have to be done.

\section{Related Work}

In cloud computing research, cloud simulators are required to evaluate the cloud system. Under different scenarios, the performance of a specific component can be evaluated by analyzing the cloud computing system [5]. For this purpose, different cloud energy consumption measuring simulators are available to measure the energy consumption of a cloud data center [19] and many researchers publish different research papers regarding their specifications. CloudSim is a Java based simulation toolkit and it can model and simulate very large-scale clouds. It can measure the energy consumed by a cloud system but less reliable than GreenCloud. It supports communication among the components of the cloud system while CloudAnalyst is derived from CloudSim [21]. On the other side CloudAnalyst is also provide java-based simulation and provide framework for deploying real-time datacenters. Its main purpose is to measure energy consumption of a cloud system [22,23]. According to the research of different researchers, GreenCloud is a packet level simulator and its ability to measure energy consumed by the system is more efficient and reliable than other simulators i.e. CloudSim and CloudAnalyst. It is specially made for energy-aware environment. GreenCloud is designed to calculate energy consumption at any particular data center components such as link, switch, gateway etc. as well as communication between the packet levels. Further, it offers to know the workload distribution in the system $[24,25]$. These simulators have to be taken for comparative study because these are specifically designed for the measurement of energy consumption in cloud computing environments. This paper aims to compare all these simulators against different parameters to show that which simulator is better than others in cloud energy consumption measurement. 


\section{Comparative Study between CloudSim, CloudAnalyst and GreenCloud}

In this part of paper, three different cloud energy consumption measuring simulators have been discussed. CloudSim simulation framework is Java based simulation toolkit which can model very large-scale clouds and support communication among the components of the cloud system [6,7]. It is event-based simulator. It has a special feature referred as federated policy. A federated cloud is a combination of several internal and external clouds utilities to meet business needs by deploying them $[8,9,10]$. The second one is CloudAnalyst derived from CloudSim. It is also event-based simulator. It has a powerful simulation framework for deploying real-time datacenters and monitoring the load balancing. So, it can be applied to determine the behavior of large-scale internet applications on cloud $[9,10,11,12,13]$. GreenCloud is the third one simulator to determine energy consumed by the system; with the ability of improving power management, it supports the researchers in exploring methods to reduce energy consumption with user friendly graphical user interface. It is a packet-level simulator, which strictly pay attention on the communication between components. This simulator based on two languages i.e. C++ and Tool Command Language (TCL) scripts $[9,10],[14,15]$. All of these simulators have their own ability to determine the energy consumption in cloud computing environments.

\section{Methodology}

To identify the best cloud energy consumption measuring simulator for the help of researchers and others, the comparative study of the mentioned simulators has been done in two ways i.e. Technical Factors and Environmental Factors, which can be seen in section V i.e. Results. For technical factors the comparative study has been done on the following parameters: Simulator Services, Programming Language, Simulator Type and Simulation Time. For environmental factors the comparative study has been done on the following parameters: Market Demand, Graphical User Interface, Performance, Robustness and Open Source. In environmental factors, for more deeply analysis, these simulators are ranked as low, average and high to determine degree of influence and at the end their combine result has to be shown.

\section{Results}

Energy consumption can be measure with each simulator but each simulator has different capacity to perform it $[18,19,20]$. The detailed comparative study between different technical factors of cloud energy consumption measuring simulators is following:

Services-CloudSim is a Java based simulation toolkit. It can model and simulate very large-scale clouds. It supports communication among the components of the cloud system while CloudAnalyst is derived from CloudSim [6,7], [9]. It has some extended features and capabilities than CloudSim like User friendly GUI, flexibility and efficient output $[8,9]$. On the other side energy-aware cloud computing data centers can get simulation environment from GreenCloud simulator to capture the detailed energy consumed in data center [8,9], [14].GreenCloud is one of the only simulators which have power saving modes i.e. DVFS (Dynamic Voltage and Frequency Scaling) and DNS (Dynamic Network Shutdown) while CloudSim and CloudAnalyst do not have this feature [9], [11], [14,15]. They are also not considering the energy which has been spent. The detailed comparison between different services provided by cloud energy consumption measuring simulators can be seen in Table 1 . 
TABle 1. Services PRovided by Cloud Energy Consumption Measuring Simulators [7,8,9,10], [13,14]

\begin{tabular}{|l|l|c|c|c|}
\hline Sr\# & \multicolumn{1}{|c|}{ Services } & CloudSim & CloudAnalyst & GreenCloud \\
\hline $\mathbf{1}$ & Provides java-based simulation & Yes & Nes & No \\
\hline $\mathbf{2}$ & $\begin{array}{l}\text { Simulation of CPU, memory, storage and } \\
\text { networking resources }\end{array}$ & No & No & Yes \\
\hline $\mathbf{3}$ & Provides computational resources & Yes & Yes & No \\
\hline $\mathbf{4}$ & $\begin{array}{l}\text { Provide framework for deploying real-time } \\
\text { datacenters }\end{array}$ & No & Yes \\
\hline $\mathbf{5}$ & Support virtualization and VM migration & No & Yes & Yes \\
\hline $\mathbf{6}$ & $\begin{array}{l}\text { Graphical output in form of tables and } \\
\text { charts }\end{array}$ & Yes & Yes & No \\
\hline $\mathbf{7}$ & Energy consumption measurement & Power Saving Modes & Yes \\
\hline $\mathbf{8}$
\end{tabular}

Programming Language- CloudSim and CloudAnalyst simulator code is written on Java while GreenCloud simulator code is written on C++ and Tool Command Language (TCL) [8,9]. See Table 2.

Simulator Type- CloudSim and CloudAnalyst simulators are event-based while GreenCloud is a packet-level simulator. So, GreenCloud simulator captures packet-level details of energy consumption which gives accurate results [8,9], [11]. See Table 2.

Simulation Time- GreenCloud simulator provides results efficiently as compare to CloudSim and CloudAnalyst [9], [11]. See Table 2.

TABle 2. TeChnical factors of Cloud Energy Consumption MEAsuring Simulators [7], [13,14]

\begin{tabular}{|l|l|l|l|l|}
\hline Sr\# & Parameters & CloudSim & CloudAnalyst & GreenCloud \\
\hline $\mathbf{1}$ & $\begin{array}{l}\text { Programming } \\
\text { Language }\end{array}$ & Java & Java & $\begin{array}{l}\text { C++/TCL (Tool Command } \\
\text { Language) }\end{array}$ \\
\hline $\mathbf{2}$ & Simulator Type & Event based & Event based & Packet level \\
\hline $\mathbf{3}$ & Simulation Time & Less Efficient & Less Efficient & Efficient \\
\hline
\end{tabular}

The detailed comparative study between different environmental factors of cloud energy consumption measuring simulators is following:

Market Demand- Market demand of GreenCloud is higher than CloudSim and CloudAnalyst due to reliable and accurate measurement of energy consumption because it captures the detailed energy consumed by a cloud system at packet level and it can update the energy consumed by a cloud system while CloudSim and CloudAnalyst can calculate the energy consumption but do not consider the energy which they have spent [16]. Due to power saving modes GreenCloud is highly demanded simulator in cloud datacenters. For detailed 
comparative study, these simulators are ranked as low, average and high to determine degree of influence, which can be seen in Table 3 and which simulator falls in which rank can be seen in Table 7.

TABLE 3. DESCRIPTION TO DETERMINE DEGREE OF INFLUENCE FOR MARKET DEMAND

\begin{tabular}{|l|l|}
\hline Rank As & Description to determine degree of influence \\
\hline Low & $\begin{array}{l}\text { Measurement of energy consumption is not reliable because it does not consider the energy which has been } \\
\text { spent }\end{array}$ \\
\hline Average & Measure energy consumption and provide frameworks for deploying real-time datacenters \\
\hline High & $\begin{array}{l}\text { Reliable and accurate measurement of energy consumption and captures the detailed energy at packet-level } \\
\text { consumed by a cloud system }\end{array}$ \\
\hline
\end{tabular}

Graphical User Interface- CloudSim simulator has a complex graphical user interface while CloudAnalyst has a user-friendly graphical user interface. On the other side, GreenCloud simulator has much user-friendly graphical user interface than other simulators [9,10]. For detailed comparative study, these simulators are ranked as low, average and high to determine degree of influence, which can be seen in Table 4 and which simulator falls in which rank can be seen in Table 7.

TABLE 4. DESCRIPTION TO DETERMINE DEGREE OF INFLUENCE FOR GUI

\begin{tabular}{|l|l|}
\hline Rank As & Description to determine degree of influence \\
\hline Low & Complex graphical user interface as usage point of view i.e. difficult for user to understand its functionalities \\
\hline Average & Somehow easy graphical user interface for the user to understand its functionalities \\
\hline High & User friendly graphical user interface to easily understand its functionalities \\
\hline
\end{tabular}

Performance- Performance wise GreenCloud simulator is much better than other simulators due to minimal simulation time [9], [14,15]. For detailed comparative study, these simulators are ranked as low, average and high to determine degree of influence, which can be seen in Table 5 and which simulator falls in which rank can be seen in Table 7.

TABle 5. Description to DETERmine degree of influence for PERFormance

\begin{tabular}{|l|l|}
\hline Rank As & Description to determine degree of influence \\
\hline Low & Take maximum simulation time to compute the results related to energy measurement \\
\hline Average & $\begin{array}{l}\text { Take average simulation time as compare to other simulators to compute the results related to energy } \\
\text { measurement }\end{array}$ \\
\hline High & Minimal simulation time to compute the results related to energy measurement \\
\hline
\end{tabular}

Robustness- GreenCloud simulator has the ability to calculate accurate results due to its ability to capture details at packet-level while other simulators have not this ability and do not consider the energy which they have spent [7], [11], [14]. For detailed comparative study, these simulators are ranked as low, average and high to determine degree of influence, which can be seen in Table 6 and which simulator falls in which rank can be seen in Table 7 . 
TABLE 6. DESCRIPTION TO DETERMINE DEGREE OF INFLUENCE FOR ROBUSTNESS

\begin{tabular}{|l|l|}
\hline Rank As & Description to determine degree of influence \\
\hline Low & Calculate the energy consumed by a cloud system but do not consider the energy which has been spent \\
\hline Average & Calculate the energy consumed by a cloud system and consider the energy which has been spent \\
\hline High & $\begin{array}{l}\text { Calculate and update the energy consumed by a cloud system and strictly pay the attention between } \\
\text { communication of different components }\end{array}$ \\
\hline
\end{tabular}

Open Source- CloudSim, CloudAnalyst and GreenCloud simulators are open source and easily available [9], [11].

The detailed comparison between different environmental factors of cloud energy consumption measuring simulators can be seen in Table 7 .

TABLE 7. ENVIRONMENTAL FACTORS OF CLOUd ENERGy CONSUMPTION MEASURING Simulators [7], [9], [13,14]

\begin{tabular}{|c|c|c|c|c|}
\hline Sr\# & Parameters & CloudSim & CloudAnalyst & GreenCloud \\
\hline 1 & Market Demand & Low & Average & High \\
\hline 2 & GUI & Low & High & High \\
\hline 3 & Performance & Low & Average & High \\
\hline 4 & Robust & Low & Low & High \\
\hline 5 & Open Source & Yes & Yes & Yes \\
\hline
\end{tabular}

It can be seen in Table 7 that the market demand of GreenCloud is higher than other simulators and its graphical user interface is also user-friendly. Its performance, simulation time and robustness are also better than other simulators. GreenCloud simulator has the ability to measure full energy consumed by each component of the cloud system because it is a packet-level simulator which captures the detailed energy while CloudSim and CloudAnalyst are event-based simulators, so they have limited ability to measure energy consumption. The advantage of GreenCloud simulator is that it can calculate and update the energy consumed by cloud system. On the other side, CloudSim and CloudAnalyst have the ability to calculate the energy but have lesser capabilities than GreenCloud [14]. GreenCloud has different power-saving modes i.e. DVFS (Dynamic Voltage and Frequency Scaling) and DNS (Dynamic Network Shutdown) while CloudSim and CloudAnalyst don't have [14,15].

\section{Conclusion}

Many researchers use cloud energy consumption measuring simulators for the testing and evaluation of cloud systems. By comparing these simulators, it can be seen that GreenCloud simulator has several benefits over CloudSim and Cloud Analyst. GreenCloud implements different power-saving algorithms and capture packet-level details. GreenCloud simulator calculates the energy consumption accurately because energy consumption is computed on each packet arrival, task execution and completion. So, GreenCloud has the ability to calculate accurate results related to energy consumed by a cloud system. As, GreenCloud has the limitation that it limits to only small data centers. So, it may be good direction for future work to propose a model that how it can be implement at a vast level in cloud computing data centers. 


\section{References}

[1] Cloud computing: A complete guide | IBM, https://www.ibm.com/cloud/learn/cloud-computing, Retrieved November 13, 2018

[2] Y. Jadeja K. Modi "Cloud computing-concepts architecture and challenges" Computing Electronics and Electrical Technologies (ICCEET) 2012 International Conference on pp. 877-880 2012.

[3] Q. Zhang L. Cheng R. Boutaba "Cloud computing: state-of-the-art and research challenges" J. internet Servo Appl. vol. 1 no. l pp. 7-18 2010.

[4] G. Sakellari G. Loukas "A survey of mathematical models simulation approaches and testbeds used for research in cloud computing" Simul. Model.Pract.Theory vol. 39 pp. 92-103 2013.

[5] X. Bai M. Li B. Chen W.- T. Tsai J. Gao "Cloud testing tools" Service Oriented System Engineering (SOSE) 2011 IEEE 6th International Symposium on pp. 1-12 2011.

[6] R. N. Calheiros R. Ranjan C. A. F. DeRose R. Buyya "Cloudsim: A novel framework for modeling and simulation of cloud computing infrastructures and services" arXivPrepr. arXiv0903.2525 2009.

[7] R. N. Calheiros R. Ranjan A. Beloglazov C. A. F. De Rose R. Buyya "CloudSim: a toolkit for modeling and simulation of cloud computing environments and evaluation of resource provisioning algorithms" Softw. Pract.Exp. vol. 41 no. 1 pp. 23-50 2011.

[8] F. Howell R. McNab "SimJava: A discrete event simulation library for java" Simul. Ser. vol. 30 pp. 51-56 1998.

[9]“The Best Open Source Cloud Computing Simulators”, https://opensourceforu.com/2016/11/best-open -source-cloud-computing-simulators/, Retrieved January 03, 2019.

[10] “Comparative Study of Simulation Tools in Cloud Computing Environment”,https://pdfs. semanticscholar.org/c66b/776b1a15086f23ff2099913075e01d6e6edb.pdf, Retrieved January 03, 2019.

[11] “MEDC_Project_Report_Bhathiya_318282”, http://www.cloudbus.org/students/MEDC_Project_Report_ Bhathiya_318282.pdf, Retrieved January 04, 2019.

[12] Ahmed, T., \& Singh, Y. (2012). Analytic study of load balancing techniques using tool cloud analyst. International Journal of Engineering Research and Applications, 2(2), 1027-1030.

[13] Mishra, R. K., \&Bhukya, S. N. (2014). Service broker algorithm for cloud-analyst. International Journal of Computer Science and Information Technologies, 5(3), 3957-3962.

[14] D. Kliazovich P. Bouvry S. U. Khan "GreenCloud: a packet-level simulator of energy-aware cloud computing data centers" J. Supercomput. vol. 62 no. 3 pp. 1263-1283 2012.

[15] L. Shang L.-S.Peh N. K. Jha "Dynamic voltage scaling with links for power optimization of interconnection networks" High-Performance Computer Archituecture 2003. HPCA-9 2003. Proceedings.The Ninth International Symposium on pp. 91-102 2003.

[16] “A review on Cloud Simulators”, https://pdfs.semanticscholar.org/1434/a866970a2338db41bca218 fcb358caaf07cc.pdf, Retrieved January 16, 2019.

[17] R. Buyya C. S. Yeo S. Venugopal J. Broberg I. Brandic "Cloud computing and emerging IT platforms: Vision hype and reality for delivering computing as the 5th utility" Futur. Gener.Comput.Syst. vol. 25 no. 6 pp. 599-616 2009.

[18] D. Kliazovich P. Bouvry S. U. Khan "Simulating communication processes in energy-efficient cloud computing systems" 2012.

[19] R. Brown "Report to congress on server and data center energy efficiency: Public law 109-431" Lawrence Berkeley Natl. Lab. 2008.

[20] X. Bai M. Li B. Chen W.- T. Tsai J. Gao "Cloud testing tools" Service Oriented System Engineering (SOSE) 2011 IEEE 6th International Symposium on pp. 1-12 2011.

[21] Shi, Yuxiang, Xiaohong Jiang, and Kejiang Ye. "An energy-efficient scheme for cloud resource provisioning based on CloudSim." 2011 IEEE International Conference on Cluster Computing. IEEE, 2011. 
[22] B. Wickremasinghe (2009), "CloudAnalyst: A CloudSim based Tool for Modeling and Analysis of Large-Scale Cloud Computing Environments”, MEDC Project Report.

[23] B. Wickremasinghe \& R.N. Calheiros (2010), "CloudAnalyst: A CloudSim based Visual Modeler for Analyzing Cloud Computing Environments and Applications”, 24th International Conference on Advanced Information Networking and Application, Pp. 446-452.

[24] Suryateja, Pericherla S. "A Comparative Analysis of Cloud Simulators." International Journal of Modern Education \& Computer Science 8.4 (2016).

[25] Malhotra, Rahul, and Prince Jain. "Study and comparison of cloudsim simulators in the cloud computing." The SIJ Transactions on Computer Science Engineering \& its Applications (2013).

[26] Malhotra, Rahul, and Prince Jain. "Study and comparison of cloudsim simulators in the cloud computing." The SIJ Transactions on Computer Science Engineering \& its Applications (2013).

\section{Author's Profile}

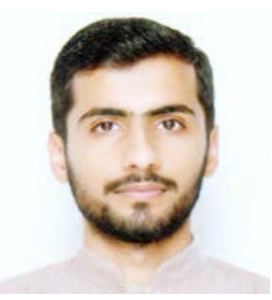

Muhammad Hassaan has completed his BS (Hons) degree in Software Engineering from University of Management and Technology, Lahore, Pakistan in 2018 and currently he is enrolled in MS Software Engineering at the same institute. Now, he is in faculty of computer science at Department of Computer Science in Virtual University of Pakistan.

How to cite this paper: Muhammad Hassaan. " A Comparative Study between Cloud Energy Consumption Measuring Simulators ", International Journal of Education and Management Engineering(IJEME), Vol.10, No.2, pp.20-27, 2020.DOI: 10.5815/ijeme.2020.02.03 